Conclusions Autoantibody profiling using 86 antigens provides an opportunity for identifying subgroups of patients with distinct marker profiles for designing clinical trials and evaluating clinical response in defined patient subgroups.

\section{DISCOVERY AND EVALUATION OF A MULTIPLEXED MASS SPECTROMETRY PANEL FOR MEASURING CANDIDATE PEPTIDE BIOMARKERS IN URINE FROM PATIENTS WITH LUPUS NEPHRITIS}

${ }^{1} \mathrm{~V}$ Anania*, ${ }^{1} \mathrm{Q} \mathrm{Li},{ }^{2} \mathrm{M}$ Cascino, ${ }^{2} \mathrm{~L}$ Dragone, ${ }^{3} \mathrm{P}$ Liu, ${ }^{3} \mathrm{~W}$ Sandoval, ${ }^{2} \mathrm{~T}$ Katsumoto, ${ }^{1}$ A Morimoto, 'A Herman, 'WR Mathews. 'Genentech, Biomarker Development, South San Francisco, USA; ${ }^{2}$ Genentech, Clinical Science, South San Francisco, USA; ${ }^{3}$ Genentech, Research Proteomics, South San Francisco, USA

\subsection{6/lupus-2017-000215.38}

Background and aims Lupus nephritis (LN) is a clinical manifestation of systemic lupus erythematosus (SLE) associated with significant morbidity and mortality. Although proteinuria is highly correlated with disease progression in LN, the composition of the LN urinary proteome remains poorly characterised. To address this issue, complementary mass spectrometry (MS)-based approaches were used to identify candidate urinary biomarkers and a targeted proteomics panel was developed to further assess levels in LN samples.

Methods LN urine samples were profiled using three MSbased methods: 2D SDS-PAGE, chemical labelling using isobaric mass tags, and data-independent acquisition (DIA). A quantitative, multiple reaction monitoring method was developed to further evaluate levels of these candidate peptide biomarkers in a larger cohort.

Results Using these discovery proteomic approaches $>2600$ proteins were identified, 290 of which are up-regulated in LN samples. While chemical labelling enabled identification of more total proteins, DIA outperformed chemical labelling in identification of proteins significantly up-regulated in LN samples. Further evaluation of a selected panel revealed increases in the majority of candidate peptide biomarkers in LN samples compared to healthy controls, including peptides from proteins involved in inflammation and adaptive immunity.

Conclusions These results indicate that peptides from proteins involved in inflammation and adaptive immunity can be quantified in urine of LN patients using a multiplexed MS-based method. Results from this study will be used to inform longitudinal and interventional studies focused on understanding the biological implications of these candidate biomarkers and to direct development of novel tools to evaluate disease progression and treatment efficacy of current and future LN therapeutics.

\section{PERIPHERAL IMMUNOPHENOTYPING IDENTIFIES THREE SUBGROUPS BASED ON T CELL HETEROGENEITY IN LUPUS PATIENTS}

S Kubo*, S Nakayamada, M Yoshikawa, Y Miyazaki, K Sakata, K Nakano, S Iwata, I Miyagawa, K Saito, Y Tanaka. University of Occupational and Environmental Health, The first department of internal medicine, Kitakyushu, Japan

\subsection{6/lupus-2017-000215.39}

Background and aims To elucidate the diversity of systemic lupus erythematosus (SLE), we stratified SLE patients based on immunophenotyping.
Methods Peripheral blood mononuclear cells were obtained from 80 active SLE patients (with one or more BILAG category $\mathrm{A}$, or two or more BILAG category $\mathrm{B}$ ). Circulating $\mathrm{B}, \mathrm{T}$ and dendritic cells were defined based on flow cytometric analysis for human immune system termed "the Human Immunology Project". Based on these results, the immunophenotype was visualised by principal component analysis and SLE patients classified into subgroups by cluster analysis.

Results Principal component analysis indicated that the immunophenotype of active SLE patients was consistent with $\mathrm{T}$ and B cell axes. Among these correlations, Th17 and Treg cells were statistically close, and showed positive correlation $(\mathrm{p}<0.001)$. Furthermore, Tfh and Th1 cells were also statistically close, and showed positive correlation $(p=0.04)$. The same pattern was also noted between Tfh and plasmablasts $(p=0.02)$. Cluster analysis showed that SLE patients were divided into three subgroups (with high proportions of plasmablasts in all groups): patients did not show any characteristic features other than increased plasmablasts ( $\mathrm{T}$ cell-independent group), patients with high percentage of Tfh cells (Tfh-dominant group), and patients with high proportions of activated Treg and memory Treg and low proportion of naïve Treg (Treg-dominant group).

Conclusions Our study indicates that SLE patients can be divided into three subgroups based on $\mathrm{T}$ cell heterogeneity. This heterogeneity should be taken into consideration not only in basic research but also in patient selection in clinical trials for development of new drugs.

\section{ELUCIDATING GENETIC PATHWAYS IN SLE AND STRATIFYING PATIENTS VIA WHOLE GENOME SEQUENCING}

${ }^{1} \mathrm{~J}$ Ellyard*, ${ }^{1} \mathrm{R}$ Jerjen, ${ }^{1} \mathrm{E}$ McEwan, ${ }^{1} \mathrm{M}$ Field, ${ }^{1} \mathrm{~V}$ Athansopoulos, ${ }^{1} \mathrm{~S}$ Jiang, ${ }^{1} \mathrm{D}$ Andrews, ${ }^{2} \mathrm{~V}$ Pascual, ${ }^{3} \mathrm{P}$ Peterson, ${ }^{4} \mathrm{P}$ Hertzog, ${ }^{5} \mathrm{~S}$ Alexander, ${ }^{1} \mathrm{M}$ Cook, ${ }^{1} \mathrm{C}$ Vinuesa. ${ }^{1}$ Australian National University, John Curtin School of Medical Research, Canberra, Australia; ${ }^{2}$ Baylor Institute for Immunology Research, Centre for Inflammation and Autoimmune Diseases, Dallas, USA; ${ }^{3}$ University of Tartu, Department of Biomedicine, Tartu, Estonia; ${ }^{4}$ Hudson Institute of Medical Research, Centre for Innate Immunity and Infectious Diseases, Clayton, Australia; ${ }^{5}$ Children's Hospital at Westmead, Centre for Kidney Research, Westmead, Australia

\subsection{6/lupus-2017-000215.40}

Background and aims Systemic lupus erythematosus (SLE) is a heterogeneous autoimmune disease. Twin studies indicate a strong genetic contribution to lupus, yet often the pathogenic variant remains unknown. A better understanding of the individual genetic causes of SLE will enable personalised therapies. Using next generation sequencing technologies (WES/WGS) it is now possible to identify rare/novel gene variants that cause disease.

Methods We have used WES/WGS to identify rare genetic variants with strong effects that contribute to SLE and complex autoimmunity. The effect of variants on protein function were evaluated using in vitro biochemical and over-expression assays. Immunophenotyping of patient PBMCs and the use of bespoke mouse models engineered by CRISPR/Cas9 to harbour patient-specific variants were used to dissect disease mechanisms.

Results We identified a genetic variant in TREX1 as a cause of cerebral SLE, providing proof of principle that rare genetic variants do contribute to complex autoimmunity. The patient was revealed to be a prime candidate for tailored therapies 